\title{
Black box audio/video recording in the operating room: time for anesthesiologists to get with the picture
}

\author{
David Levin, MD $\cdot$ Stefanie Tan, MSc
}

Received: 12 March 2015 / Accepted: 25 March 2015/Published online: 31 March 2015

(c) Canadian Anesthesiologists' Society 2015

\section{To the Editor,}

I recently spent time at a hospital where the surgical department initiated research efforts to evaluate how audio/ video $(\mathrm{A} / \mathrm{V})$ recording in the operating room $(\mathrm{OR})$ - akin to black boxes in the aviation industry - can be used to improve quality of care through review of critical incidents. However, this workplace surveillance may have ramifications that extend beyond the insights gained from the original objective to review $\mathrm{A} / \mathrm{V}$ footage after critical incidents. Surgeons have generated most of the published evidence and opinions available on this topic ${ }^{1,2}$ with little participation or input from the anesthesiology community. Even so, anesthesiologists have a vested interest in the pursuit of this type of policy, both as experts in delivering perioperative care, and importantly, because they can become the subjects of $\mathrm{A} / \mathrm{V}$ footage.

In addition to the parallels in safety improvement drawn from the aviation industry's use of black boxes, proponents of routine $\mathrm{OR} \mathrm{A} / \mathrm{V}$ recordings also draw comparisons to the wearable cameras employed by some police forces. However, police forces were motivated to install wearable cameras to improve accountability by influencing real-time behavioural improvements in light of critical lapses in professionalism with regard to racial profiling and abuse of force. ${ }^{3}$ As routine $\mathrm{A} / \mathrm{V}$ recordings can motivate both positive and negative behavioural

D. Levin, MD $(\bowtie)$

Department of Anesthesia, University of Toronto,

Toronto, ON, Canada

e-mail: david.levin@utoronto.ca

S. Tan, MSc

Policy Innovation Research Unit, Department of Health Services Research and Policy, London School of Hygiene and Tropical Medicine, London, UK changes in real time, it is possible that workplace surveillance could increase the potential for litigation and non-allowances for normal practice variations as well as create a culture of overly cautious practice.

There is a growing view that improving quality and safety should be a more dominant aspect of the medical culture. This is motivated not only by the increased recognition that medical error plays a large role in morbidity and mortality but also by a widely publicized catastrophic lapse in professionalism that incited a call for greater means to enforce accountability. A particularly striking incident occurred in Toronto, Ontario where an anesthesiologist was convicted of sexual abuse that took place inside the OR. ${ }^{4}$ In Ontario, events like this have sparked the development of a task force to review legislation to prevent sexual abuse of patients. ${ }^{5}$ It is clear that routine $\mathrm{A} / \mathrm{V}$ recording could be an influential tool for proponents of such legislation.

The use of $\mathrm{A} / \mathrm{V}$ recording in the OR (and potentially in other locations in the hospital) raises many questions. For example, how will patients respond? Would patients or staff be able to limit the scope of information captured? Will recordings become part of the medical record? Would physicians be responsible for routinely reviewing the files? Will the recordings become a tool to generate punitive incentives? Will the routine use of the recordings make physicians less likely to proceed with higher-risk procedures or patients? The possibility of it becoming a perverse policy tool will require careful consideration before introduction into clinical practice.

Black box recordings in the OR have the potential to improve but also to limit anesthesia practice. With careful implementation and strong regulatory measures, this technology could potentially improve care, but there are some major issues and motivations that need to be carefully 
considered. We advocate for anesthesiologists to take a more active role in this emerging process.

Funding The authors did not receive any funding to support this work.

Commercial or non-commercial affiliations The authors have no conflicts of interest to declare.

Other associations The authors have no other associations or consultancies to declare.

\section{References}

1. Sade RM, American Association for Thoracic Surgery Ethics Committee; Society of Thoracic Surgeons Standards and Ethics Committee. Broadcast of surgical procedures as a teaching instrument in cardiothoracic surgery. Ann Thorac Surg 2008; 86: 357-61.

2. Millat B, Fingerhut A, Cuschieri A. Live surgery and video presentations: seeing is believing ... but no more: a plea for structured rigor and ethical considerations. Surg Endosc 2006; 20: 845-7.

3. Mims C. What Happens When Police Officers Wear Body Cameras. The Wall Street Journal 2014.

4. Jones A. Toronto Doctor Who Sexually Assaulted Patients During Surgeries Sentenced to 10 Years - 2014. Available from URL: http://news.nationalpost.com/2014/02/25/toronto-doctor-who-sexuallyassaulted-patients-during-surgeries-sentenced-to-10-years/ (accessed March 2015).

5. Ministry of Health and Long-Term Care. Newsroom: New Minister's Task Force to Review Legislation to Prevent Sexual Abuse of Patients - 2014. Available from URL: http://news. ontario.ca/mohltc/en/2014/12/new-ministers-task-force-to-reviewlegislation-to-prevent-sexual-abuse-of-patients.html (accessed March 2015). 